\title{
Contrôle génétique de la squelettogenèse
}

\section{Patricia Ducy}

\section{ADRESSE}

P. Ducy: Department of Molecular and Human Genetics and Department of Medicine, Baylor College of Medicine, One Bay-
Les progrès de la génétique ont permis d'établir avec précision au cours de ces dix dernières années la succession des événements moléculaires et cellulaires présidant à la mise en place du squelette. Dans l'embryon, les bourgeons squelettiques s'individualisent à partir de trois régions: le mésoderme de la plaque latérale, les somites et les cellules des crêtes neurales. Cette spécification des cellules mésenchymateuses est sous le contrôle de facteurs dits «organisateurs » FGF, BMP, ou Ihh dont l'altération conduit à des troubles de la forme générale du squelette. Ces ébauches évoluent ensuite indépendamment, et suivent deux voies d'ossification très distinctes: ossification dite membranaire, où les cellules mésenchymateuses se différencient in situ en ostéoblastes; ossification endochondrale, où une population centrale de chondrocytes se différencient et s'organisent en une plaque de croissance cartilagineuse, primordiale pour la croissance longitudinale des os. Chacun de ces processus d'ossification est très étroitement contrôlé par des facteurs de transcription (Sox-9, cbfa1), des facteurs de croissance et leurs récepteurs (FG FR3, PT H rP), chacun intervenant à une étape très précise de la cascade moléculaire assurant la différenciation chondrocytaire et ostéoblastique. L'altération de la fonction de ces signaux, conséquence de mutations souvent activatrices, entraîne des anomalies du squelette correspondant à des maladies humaines. 
clastes dans la partie ossifiée des os, sont maintenant identifiés. Plus important encore, au-delà de ces connaissances restées longtemps fragmentaires, il devient maintenant possible d'établir une cascade génétique intégrant la fonction de ces différents facteurs aux événements cellulaires et structuraux qui président à la formation des éléments du squelette. Cet article se propose de faire le point sur ces récentes avancées et de présenter la vision actuelle des étapes et des régulations génétiques majeures participant au développement embryonaire du squelette.

\section{Morphogenèse des futurs éléments squelettiques}

L'étude du processus initial de morphogenèse des futurs os occupe à elle seule une branche particulière de la biologie du développement. Plusieurs revues récentes ont fait un point détaillé sur les dernières avancées de cette discipline [1, 2]. Nous nous limiterons donc à mettre en valeur certains des principes généraux caractérisant ce processus initial en les illustrant d'exemples représentatifs.

A l'instar de la plupart des autres processus organogénétiques, la formation du squelette débute par la convergence et la condensation de cellules en majorité mésenchymateuses en lieu et forme de chacun de ses futurs éléments. Trois régions de l'embryon vont être à l'origine du squelette. Les membres dérivent du mésoderme de la plaque latérale, le squelette axial (colonne vertébrale et cage thoracique) dérive des somites et le squelette craniofacial est majoritairement formé par des cellules originaires des crêtes neurales [3]. Les facteurs qui contrôlent cette première étape jouent donc un rôle d'organisateur à l'échelle de l'embryon et cette fonction sera plus particulièrement illustrée par le type d'anomalies que leur absence engendre. En effet, l'absence de ces facteurs se traduit par une altération plus ou moins étendue de la forme générale du squelette car elle entraîne l'absence, la malformation ou l'apparition ectopique d'un ou plusieurs éléments squelettiques. $\mathrm{Ce}$ type de phénotype est différent de celui qu'engendre la déficience en un facteur de différenciation cellulaire (voir plus loin): dans ce cas, la forme générale du squelette est préservée mais un type de cellules reste bloqué à un stade donné de la différenciation, incapable de synthétiser les molécules structurales nécessaires à la constitution ou à la fonction d'os mûrs.

Le processus de morphogenèse initiale est orchestré par des facteurs de croissance principalement du type FGF (fibroblast growth factor), Hedgehog ou BMP (bone morphogenetic protein) [3]. D'une façon générale, ces facteurs règlent souvent des processus qui ne se limitent pas au squelette, organisant par exemple la structure générale d'une ou de plusieurs régions de l'embryon. Ainsi, I'inactivation chez la souris du gène codant pour le FGF10 aboutit à la production de souris sans membres [4]. Ces facteurs peuvent aussi contrôler des processus morphogénétiques totalement distincts de la squelettogenèse. Dès lors, leur absence chez l'homme ou chez la souris entraînera des anomalies souvent létales car affectant à la fois le squelette et la formation d'un ou de plusieurs autres organes vitaux. Ainsi, les souris déficientes en plusieurs des BMP n'atteignent même pas le stade embryonnaire qui marque le début de la squelettogenèse [5]. Par exemple, les souris B mp4 $\%$ meurent entre les jours E6.5 et E9.5 de l'absence d'individualisation du mésoderme, et celles privées de Bmp2 meurent avant le jour E10 car la morphogenèse du canal préamniotique puis celle du cœur est défectueuse [5].

Au niveau transcriptionnel, la morphogenèse des condensations mésenchymateuses semble généralement contrôlée par les facteurs de transcription à homéoboîte, notamment des sous-familles Hox, Pax et Msx [3]. Le plus souvent, chacun de ces facteurs ne contrôle la mise en place que d'une fraction limitée d'éléments squelettiques, et son absence provoquera des dysostoses (modification d'un os ou d'un nombre restreint d'os) plutôt que des dysplasies (altérations généralisées sur tout le squelette). Par exemple, la mutation du gène codant pour Hoxd-13 engendre une syn polydactylie (fusion et duplication des doigts) chez I'homme comme chez la souris [1].
De même, l'inactivation de Msxl cause les mêmes anomalies chez ces deux espèces: absence de fermeture du palais, malformation des os du crâne, du nez et de l'oreille interne, absence de développement dentaire $[6,7]$. L'analyse du rôle spécifique de ces facteurs est fréquemment compliquée par la co-expression de molécules de la même famille au sein de certains tissus et la redondance de leur fonction. Ainsi, les facteurs de transcription à paired-box, Pax 1 et Pax9, sont tous deux exprimés dans le sclérotome, c'est-à-dire la région ventromédiale des somites qui donnera naissance aux vertèbres, et comme leur domaine de liaison à I'ADN est conservé à $98 \%$, ils peuvent se lier aux mêmes promoteurs cibles et y remplir la même fonction. De ce fait, les con séquences de l'inactivation de Pax1 ou de Pax9 sur la formation du squelette axial sont limitées dans le premier cas, inexistantes dans le second. En revanche, I'inactivation simultanée des deux gènes empêche la formation des disques vertébraux comme intervertébraux [8]. De la même façon, la co-expression et la redondance fonctionnelle des facteurs appartenant à famille Msx ont compliqué l'établissement de leur rôle dans la morphogenèse craniofaciale. Alors que les souris déficientes soit en Msx1 [6], soit en Msx2 [9], présentent seulement des malformations de quelques éléments osseux, celles où les deux gènes ont été inactivés sont totalement dépourvues de toute la partie supérieure du crâne et de la majeure partie des mâchoires [9], démontrant clairement le rôle critique que ces facteurs jouent dans leur formation.

A l'évidence, les facteurs d'organisation des condensations squelettiques, ou tout au moins certains d'entre eux, vont éventuellement déclencher l'expression du groupe suivant de régulateurs, qui président à la différenciation des cellules. A I'heure actuelle, peu de données démontrent la réalité d'une telle cascade aux niveaux génétique et moléculaire, c'est-à-dire apportent la preuve directe d'une régulation transcriptionnelle. II existe trois explications à cette relative absence d'informations: (1) les facteurs clés contrôlant la différenciation des chondrocytes ou des ostéoblastes n'ont été identi- 
fiés que très récemment alors que les nombreuses études sur la morphogenèse sont antérieures. Une analyse complémentaire du phénotype de certains modèles de souris mutantes, antérieurs aux 4 dernières années, pourra remédier à ce problème; (2) beaucoup de ces mutants ayant des phénotypes complexes et létaux, il faudra probablement attendre la production de nouveaux modèles d'inactivation conditionnelle des gènes (soit tardive, soit spécifique du squelette) pour détecter certaines modifications d'expression pertinentes; (3) enfin, troisième pierre d'achoppement, chez tout mutant, il est difficile de faire la distinction entre l'absence d'expression d'un facteur de différenciation et l'absence ou la transformation des cellules qui seraient supposées l'exprimer. Pour prouver qu'un facteur morphogénétique particulier règle directement l'expression d'un facteur de différenciation, il faudra vraisemblablement attendre la caractérisation des régions régulatrices présentes dans le promoteur de ce dernier.

Chacune des condensations mésenchymateuses, une fois formées, va évoluer pour son propre compte vers la formation d'un élément ossifié, indépendamment de son origine ou de sa position. On distingue deux processus distincts d'ossification, l'ossification endochondrale et l'ossification membranaire, décrits séparément, en insistant sur les mécanismes de régulation démontrés in vivo chez la souris et ayant une correspondance avec des maladies humaines.

\section{Contrôle génétique de l'ossification intramembranaire}

Quelques structures, dont la plupart des os plats du crâne et la partie latérale des clavicules par exemple, se forment selon un processus où les cellules mésenchymateuses indifférenciées prolifèrent d'abord au sein de la condensation puis se différencient in situ et directement en ostéoblastes. C'est le processus d'ossification membranaire. Dans ce cas, faire la distinction entre un phénotype causé par l'absence d'un morphogène et celui causé par l'absence d'un facteur de différenciation cellu- effet, I'absence de formation des condensations, l'absence de prolifération cellulaire en leur sein, et l'impossibilité des cellules de se différencier en ostéoblastes, causeront toutes une absence des os d'origine intramembranaire. En fait, seules des études poussées analysant spécifiquement le taux de prolifération et/ ou d'apoptose des cellules, de même que leur capacité d'exprimer ou non certains marqueurs des cellules différenciées ou non, permettent d'établir une distinction exacte entre ces trois origines. Alternativement, quand un facteur joue un rôle dans les deux types d'ossification, intramembranaire et endochondrale, il est plus facile de déterminer s'il appartient au groupe des organisateurs ou à celui des différenciateurs. Le facteur de transcription Cbfal est un exemple représentatif de cette catégorie de régulateurs. Chez la souris, son absence bloque toute possibilité de différenciation ostéoblastique. Au niveau du crâne, elle cause la persistance de la structure membranaire formée par les condensations mésenchymateuses jusqu'à la naissance [10], période au cours de laquelle les mutants homozygotes meurent (voir plus loin). Ce phénotype, associé à l'absence de clavicules, formées également par ossification intramembranaire chez la souris, est causé par l'absence complète de différenciation des celIules mésenchymateuses en ostéoblastes fonctionnels, seules cellules capables de sécréter une matrice osseuse. Comme dans le cas de l'ossification intramembranaire, aucun moule cartilagineux n'est déposé avant la formation d'une matrice osseuse (voir plus loin, ossification endochondrale), aucune structure matricielle ne vient imposer sa cohésion à l'intérieur des condensations mésenchymateuses et le résultat est l'absence de structure visible par les méthodes usuelles de mise en évidence du squelette. Dans ce cas, l'absence totale d'une structure n'est pas le reflet d'une morphogenèse défaillante mais plutôt celui d'une absence de différenciation cellulaire. En deçà de la difficulté de classification des phénotypes et des facteurs leur correspondant, il existe une raison biomédicale qui a déterminé l'orientation de la majeure partie des études réalisées sur l'ossification intramembranaire. Parce que plus d'un nouveau-né sur 2500 présente une malformation crânienne due à une fusion accélérée ou ralentie des os plats crâniens [11], la plupart des études ont été centrées sur l'étude non pas de la formation des ces os mais de la dynamique de formation et d'ossification des sutures les séparant. Les os du crâne grandissent dans un premier temps de façon centrifuge puis se rejoignent finalement au niveau de régions appelées sutures (figure 1). Tant que les os grandissent, ces sutures ne s'ossifient pas afin que l'élargissement de la boîte crânienne s'adapte à la croissance du cerveau. Une fois la taille finale de ce dernier atteinte, les sutures vont s'ossifier et les os fusionner. Quand cette fusion se fait prématurément, il s'ensuit une craniosynostose qui se manifeste par une réduction/déformation de la taille de la boîte crânienne et dont la sévérité dépend de la précocité de la fusion. A l'inverse, un retard de croissance des os ou de la capacité d'ossification des sutures entraîne la persistance post-natale d'espaces entre certains os. L'étude de plusieurs maladies génétiques humaines présentant l'une ou l'autre de ces anomalies a permis l'identification de facteurs contrôlant ce processus.

La soudure précoce des sutures coronales est notamment observée dans les syndromes de type Crouzon, Apert, Pfeiffer et Jackson-Weiss (Tableau I). Dans toutes ces maladies, des mutations dominantes activatrices ont été détectées chez les membres du sytème FGF/ FGF récepteur (FGFR) (pour revue, voir [11]), suggérant que cette voie de signalisation favorise l'ossification des sutures. La mutation correspondant au syndrome de Pfeiffer (Pro250Arg) a été reproduite chez la souris, où elle induit le même phénotype que chez I'homme, et où la nature activatrice de la voie de signalisation FGF/ FGFR a pu être confirmée au niveau moléculaire [12]. Le syndrome de Saethre-Chotzen (acrocéphalodactylie type (II), qui se caractérise aussi par une fusion prématurée des sutures coronales, est causé par la présence d'une mutation inactivant l'un des deux allèles codant pour Twist, un 


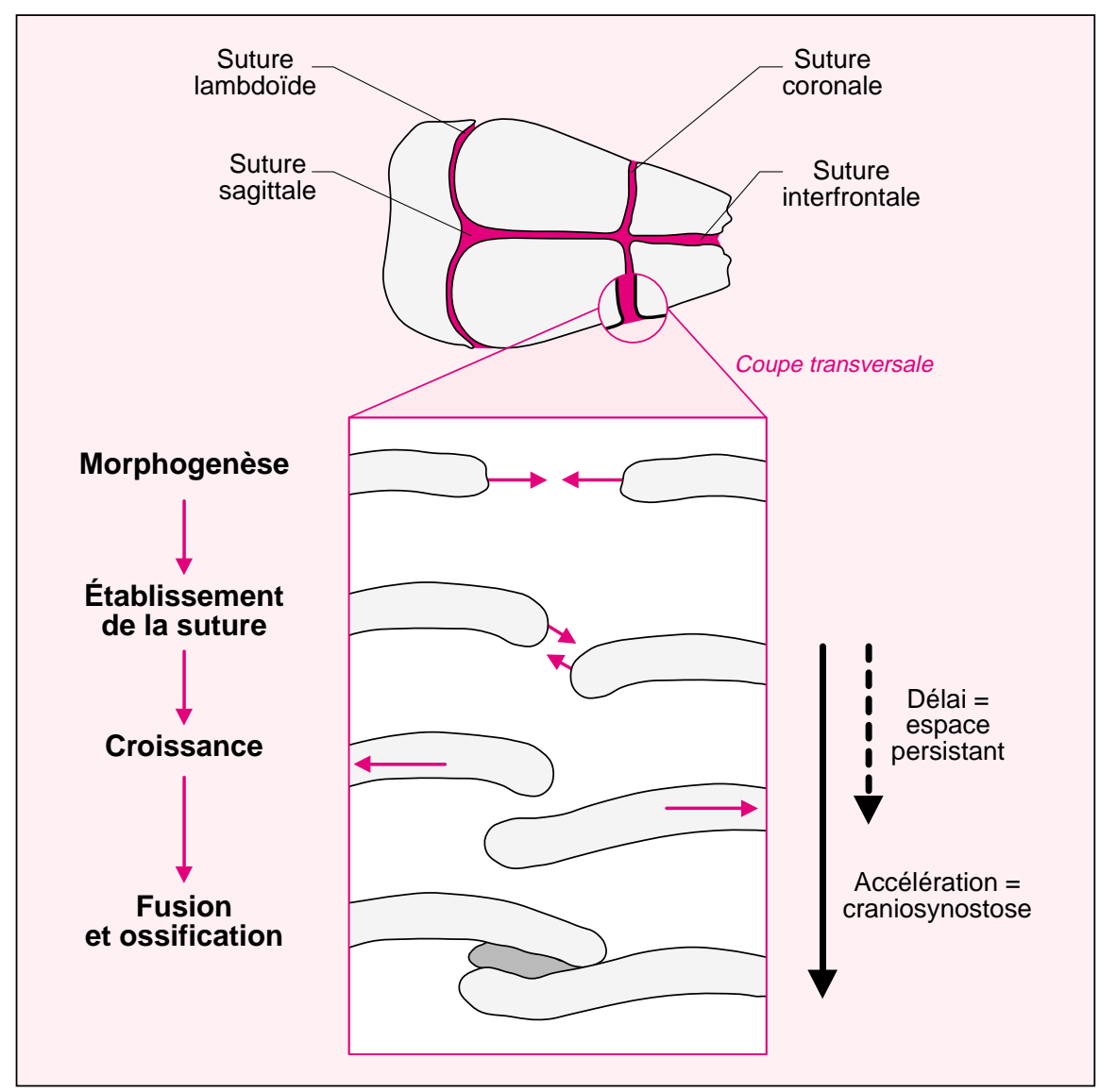

Figure 1. Dynamique des sutures crâniennes. Chacun des os plats du crâne (en gris clair) se formant par ossification intramembranaire commence par s'étendre de façon centrifuge par rapport à son futur point central. Sitôt que deux os en formation se rejoignent, leurs fronts émettent des signaux qui dévient leur course et établissent un espace non ossifié entre eux, la suture. Les os grandissent ensuite de façon centrifuge par rapport à cette suture. Une fois leur croissance achevée, les fronts des deux os fusionnent et la suture s'ossifie (gris foncé).

facteur de transcription de la famille des bHLH (basic helix loop helix) [13, 14] (voir p. 1281 de ce numéro). Ce résultat implique que Twist agit comme un inhibiteur de l'ossification des sutures. Le même phénotype existe chez les souris Twist atteintes d'haplo-insuffisance chez lesquelles il a été corrélé avec une expression ectopique du FGFR2 [13, 15]. Or, chez la drosophile, Twist contrôle l'expression de l'un de ces récepteurs, suggérant l'existance d'une cascade génétique dans laquelle T wist réglerait négativement l'expression des récepteurs des FGF, inducteurs de la fusion des sutures. La craniosynostose de type Boston est quant à elle un syndrome très rare causé par une mutation activatrice du facteur de transcription M sx2 $\mathrm{m} / \mathrm{s} \mathrm{n}^{\circ} 12$, vol. 17, décembre 2001

\section{Contrôle génétique de l'ossification endochondrale}

L'ossification de type endochondral est prépondérante chez les vertébrés supérieurs. En effet, tout le squelette appendiculaire (os longs des membres) et axial se forme selon ce processus. Contrairement à l'ossification intramembranaire, le développement endochondral comporte plusieurs étapes aussi bien au niveau génétique, cellulaire, que tissulaire. Au cours des 5 dernières années, notre connaissance de ces différentes étapes, comme des mécanismes moléculaires contrôlant leur enchaînement, a fait des progrès remarquables. Le déroulement de ce processus est schématisé dans la figure 2.

\section{Première étape : production d'un modèle cartilagineux}

Une fois chaque élément préfiguré par une condensation, les cellules la composant vont progressivement acquérir une forme différente selon leur position. En périphérie, elles vont prendre un aspect allongé et s'organiser en plusieurs couches serrées. Cette structure lamellaire sera à l'origine du périchondre et de la structure primitive entourant la région médiane des futurs os, appellée bone collar, dont les cellules se différencieront ultérieurement en ostéoblastes. A l'inverse, les cellules du centre de la condensation vont s'arrondir et s'entourer d'une matrice abondante, riche en protéoglycanes et en collagène de type II. Cette morphologie, comme la capacité de sécréter ce type de matrice extracellulaire, sont les manifestations d'une différenciation en chondrocytes. Cette première étape de ségrégation cellulaire est sous le contrôle de Sox9, un facteur de transcription de la famille HMG (high mobility group). L'importance de Sox9 dans la formation des cartilages primordiaux a été mise à jour par I'identification de mutations dans le gène humain $50 \times 9$, chez des patients atteints d'une malformation létale dominante des cartilages, nommée dysplasie campomélique [18]. Une explication plus moléculaire de ce rôle est apparue par la suite avec 


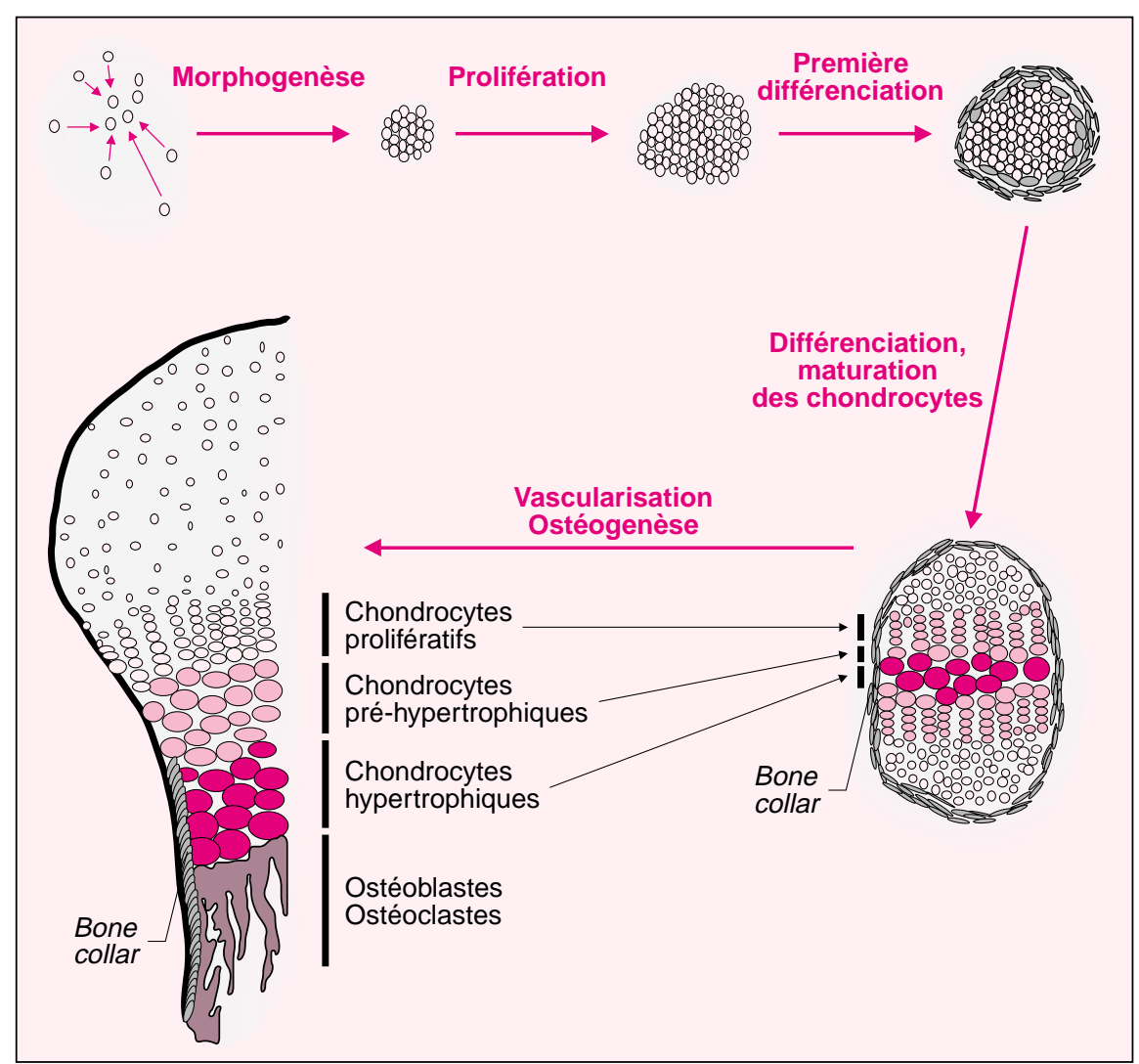

Figure 2. Représentation schématique des différentes étapes de l'ossification endochondrale. Des cellules indifférenciées migrent et s'agrègent pour former les condensations à l'origine de chaque élément squelettique. Après une phase de prolifération, les cellules des condensations subissent une première différenciation. Tandis que les cellules situées en périphérie s'aplatissent et s'organisent en une multi-couche qui sera à l'origine du «bone collar», celles situées au centre se différencient en chondrocytes synthétisant une matrice cartilagineuse. Une seconde phase de différenciation suit, lors de laquelle les chondrocytes deviennent prolifératifs puis hypertrophiques, puis apoptotiques. Sous l'influence de facteurs sécrétés par les chondrocytes matures, les ostéoprogéniteurs du «bone collar» se différencient en ostéoblastes et pénètrent au niveau de la zone hypertrophique en même temps que celle-ci est vascularisée. Les ostéoclastes, dont les progéniteurs sont amenés par la circulation sanguine, résorbent la matrice cartilagineuse qui est alors remplacée par une matrice osseuse déposée par les ostéoblastes différenciés.

I'identification de Sox9 comme l'un des facteurs de transcription contrôlant l'expression spécifique du gène de collagène de type II dans les chondrocytes [18]. Enfin, plus récemment, I'analyse de souris chimères comportant des cellules Sox $9 \%$ a montré que seules des cellules exprimant Sox9 peuvent être à l'origine des chondrocytes primordiaux formant le centre des modèles cartilagineux [19].

Deux autres membres de la famille HMG, L-Sox5 et Sox6, jouent également un rôle prépondérant dans la
Leur importance a également été mise en évidence par l'étude du site conférant au gène de collagène de type II sa spécificité d'expression dans les chondrocytes. Cependant, à la différence de Sox9, L-Sox5 et Sox6 ne contiennent pas de domaine de transactivation, et leur rôle serait de courber I'ADN afin de faciliter I'accès, la liaison ou l'activité de Sox9 ou d'autres facteurs de transcription. L'analyse très récente des souris déficientes en l'un et l'autre a montré que leur rôle n'en est pas moins important. En effet, si chez ces souris les condensations mésenchymateuses se forment normalement, la prolifération puis la différenciation des chondrocytes primordiaux y sont considérablement ralenties, entraînant une quasi-absence de dépôt de la matrice cartilagineuse [20].

\section{Seconde étape: différenciation des chondrocytes hypertrophiques et vascularisation}

Cette seconde étape va mettre en place la plaque de croissance, une structure cartilagineuse qui persistera jusqu'à la puberté chez l'homme et dont la fonction est primordiale pour la croissance longitudinale des os (figure 2). Dans un premier temps, les chondrocytes présents dans la partie la plus médiane des condensations vont rapidement proliférer, formant des colonnes de cellules juxtaposées. Cette région est appelé zone proliférative. Les cellules situées à la partie inférieure des colonnes vont ensuite s'élargir et leur production de collagène de type II commencer à décroître. Ces cellules sont dites préhypertrophiques. S'élargissant encore, elles vont ensuite cesser de synthétiser ce type de collagène et lui substituer le collagène de type $X$. Ce changement marque le dernier stade de différenciation des chondrocytes, qui sont alors dénommés chondrocytes hypertrophiques. Enfin, les chondrocytes hypertrophiques présents dans la partie la plus centrale vont mourir suivant un processus apparemment différent de l'apoptose conventionnelle, mais encore mal connu. La galectine 3 , une molécule pouvant se lier au $\beta$-galactose, s'oppose à cette mort cellulaire puisque son absence accélère le processus [21].

Les deux formes les plus sévères et fréquentes d'ostéochondrodysplasies - I'achondroplasie et la dysplasie thanatophorique - sont associées à des mutations dans le gène codant pour le FGFR3, et cette observation a apporté l'un des premiers éléments génétiques de notre compréhension moléculaire de la maturation des chondrocytes [22]. Comme lors du contrôle de la fusion des sutures crâniennes, toutes ces mutations sont dominantes et activatrices. Elles provoquent I'activation constitutive du récepteur FGFR3 et il a été démontré que plus cette activation indépendante du ligand était forte plus le 
Tableau I. Facteurs impliqués dans des anomalies héréditaires du squelette.

\begin{tabular}{|c|c|c|c|}
\hline Facteur & Type de mutation & Maladie humaine & Présentation clinique générale \\
\hline \multicolumn{4}{|c|}{ Facteurs de transcription } \\
\hline TWIST & Haplo-insuffisance & $\begin{array}{l}\text { Acrocéphalodactylie de type III } \\
\text { (syndrome de Saethre-Chotzen) }\end{array}$ & $\begin{array}{l}\text { - craniosynostose } \\
\text { - brachydactylie et syndactylie cutanée }\end{array}$ \\
\hline \multirow[t]{2}{*}{ MSX2 } & Suractivation & Mutation de type Boston & - craniosynostose \\
\hline & Haplo-insuffisance & Foramina pariétale & - délai de fusion des sutures crâniennes \\
\hline CBFA1 & Haplo-insuffisance & Dysplasie cléidocrânienne & $\begin{array}{l}\text { - délai de fusion des sutures crâniennes } \\
\text { - hypoplasie des clavicules }\end{array}$ \\
\hline SOX9 & Haplo-insuffisance & Dysplasie campomélique & $\begin{array}{l}\text { - hypoplasie létale des cartilages } \\
\text { - féminisation }\end{array}$ \\
\hline \multicolumn{4}{|c|}{ Récepteurs de facteurs de croissance } \\
\hline FGFR1 & Suractivation & Syndrome de Pfeiffer & $\begin{array}{l}\text { - craniosynostose } \\
\text { - anomalies des doigts et des pieds }\end{array}$ \\
\hline \multirow[t]{4}{*}{ FGFR2 } & Suractivation & Syndrome de Pfeiffer & $\begin{array}{l}\text { - craniosynostose } \\
\text { - élargissement des pouces } \\
\text { et des orteils }\end{array}$ \\
\hline & & Syndrome de Crouzon & - craniosynostose \\
\hline & & Syndrome de d'Apert & $\begin{array}{l}\text { - craniosynostose } \\
\text { - syndactylie des mains et des pieds }\end{array}$ \\
\hline & & Syndrome de J ackson-Weiss & $\begin{array}{l}\text { - craniosynostose } \\
\text { - élargissement des gros orteils }\end{array}$ \\
\hline \multirow[t]{3}{*}{ FGFR3 } & Suractivation & Syndrome de Muenke & - craniosynostose coronale \\
\hline & & Achondroplasie & - hypoplasie des cartilages (nanisme) \\
\hline & & Dysplasie thanatophorique & - hypoplasie létale des cartilages \\
\hline \multirow[t]{2}{*}{ PPR* } & Absence & $\begin{array}{l}\text { Chondrodysplasie de } \\
\text { type Bloomstrand }\end{array}$ & - nanisme \\
\hline & Suractivation & $\begin{array}{l}\text { Chondrodysplasie de } \\
\text { type J ansen }\end{array}$ & - nanisme \\
\hline
\end{tabular}

* PPR: récepteur PTH/PTHrP.

phénotype induit était sévère [23]. Les mutations activatrices du FGFR3 entraînent un raccourcissement des os, principalement détectable au niveau des membres, dû à une réduction de la longueur des colonnes cellulaires de la zone proliférative. II faut remarquer que la surexpression de FGF in vivo produit un phénotype similaire [24, 25]. En revanche, chez les souris déficientes en FGFR3, la plaque de croissance est élargie par une augmentation considérable du nombre de chondrocytes prolifératifs expliquant l'accroissement de taille souris [26, 27]. La production de modèles murins surexprimant ou reproduisant certaines des mutations activatrices présentes chez l'homme a permis d'analyser la fonction de la voie de signalisation du FGFR3 à un niveau moléculaire. II a notamment été montré, qu'après la naissance, la prolifération des chondrocytes est ralentie chez ces mutants et qu'il y a une augmentation de la localisation nucléaire de Stat1 [28], un facteur de transcription activé par FGFR3 [29, 30], induisant justement I'expression de plusieurs inhibiteurs du cycle cel- lulaire dont p21 WAF/CIP. A l'inverse, durant l'embryogenèse, il semble que la prolifération est accélérée dans les chondrocytes mutants tandis que leur maturation est ralentie [31-33].

Deux autres facteurs sécrétés, PTH rP (parathyroid hormone related peptide) et Ihh, vont jouer un rôle prépondérant dans l'équilibre entre la vitesse de prolifération des chondrocytes et leur maturation. Les études génétiques qui ont permis de décrypter avec autant de précision les fonctions et les interactions des chondrocytes sont particulièrement complexes et émanent de 
l'étude d'au moins une douzaine de modèles de souris déficientes, transgéniques ou chimères. PTH rP est un facteur sécrété par les chondrocytes de la partie la plus distale de l'élément squelettique, c'est-à-dire la région qui forme ou formera le périchondre de I'os. Les souris déficientes en PTH rP ont des membres courts, morphologie caractéristique d'une chondrodysplasie [34]. Ce phénotype est dû à une réduction de la zone proliférative associée à une augmentation de la vitesse de maturation des chondrocytes hypertrophiques. Les souris surexprimant PTHrP dans les chondrocytes présentent également des membres courts mais cette fois en raison d'un ralentissement considérable de la différenciation des chondrocytes hypertrophiques [35]. Ces résultats indiquent que PTHrP agit à la fois comme un activateur de la prolifération et un inhibiteur de la différenciation terminale des chondrocytes. Le récepteur de PTHrP, PPR (PTH/PTH rP receptor) est exprimé à bas niveau par les chondrocytes prolifératifs et à haut niveau par les chondrocytes pré-hypertrophiques et les ostéoblastes [36, 37]. O utre PTH rP, PPR se lie à la PTH (parathyroid hormone), qui agit positivement sur les ostéoblastes différenciés [38], ce qui a compliqué l'interprétation des phénotypes causés par son inactivation ou sa suractivation [39]. Ces deux anomalies fonctionnelles sont respectivement la cause, en pathologie humaine, des chondrodysplasies de type Blomstrand [40, 41] et Jansen [42], mais c'est leur introduction dans des modèles murins classiques ou chimères qui a permis la meilleure compréhension du rôle de ce récepteur. En accord avec la fonction de PTH rP, l'activation constitutive de PPR [43] induit un retard de conversion des chondrocytes prolifératifs en chondrocytes hypertrophiques tandis que son absence cause une accélération de ce processus [36]. A noter que, comme dans le cas de PTH rP, activation constitutive et inactivation vont aboutir au même résultat phénotypique - la diminution de la taille des os - ce qui illustre bien l'importance de l'équilibre entre prolifération et différenciation des chondrocytes pour l'ossification endochondrale. L'analyse de souris
$P P R^{-1-}$ au sein d'une majorité de celIules normales [44] a montré que les chondrocytes déficients mûrissent si rapidement qu'ils peuvent être encore localisés au-dessus de la zone de prolifération quand ils adoptent ce phénotype. Signes moléculaires de cette différenciation précoce, ces cellules expriment le collagène de type $X$, un marqueur des chondrocytes hypertrophiques, ou Ihh, un marqueur des chondrocytes pré-hypertrophiques.

C'est à l'origine grâce à des expériences d'expression ectopique chez l'embyon de poulet qu'il a été démontré que l'expression d'Ihh par les chondrocytes pré-hypertrophiques prévient leur hypertrophie en induisant la production de PTHrP par les cellules du périchondre [37]. L'analyse des souris déficientes en Ihh a permis de confimer cette boucle de régulation car PTHrP n'y est pas exprimé et il y existe une maturation anormale des chondrocytes [45]. De plus, en accord avec un phénotype plus sévère que celui des souris PT H rP-1-, l'étude des souris $\mathrm{Ihh}^{-1-}$ a mis en évidence d'autres fonctions d'Ihh dans l'ossification endochondrale. Ainsi, leur étude a notamment montré qu'lhh contrôle la prolifération des chondrocytes avant le déclenchement de leur différenciation, selon un mécanisme indépendant du système PTH rP/ PPR [ 45, 46].

On a récemment mis en évidence que le facteur de transcription Cbfal réglait positivement la différenciation hypertrophique des chondrocytes $[47,48]$. Au cours du développement, Cbfal est initialement exprimé dans toutes les cellules de la condensation squelettique en voie de maturation, puis son expression se restreint aux chondrocytes pré-hypertrophiques et aux cellules formant le bone collar [10,47]. Outre I'absence de différenciation ostéoblastique (voir plus loin), la déficience en Cbfal cause un blocage de la maturation des chondrocytes dans certains éléments squelettiques [10]. La restauration de l'expression de Cbfal dans les chondrocytes pré-hypertrophiques par un transgène supprime ce blocage, permettant l'expression d'Ihh, et la poursuite normale de la maturation des chondrocytes vers I'hypertrophie [47]. A noter que tan- dis que la boucle de régulation PTH rP/ Ihh se perpétue après la naissance et jusqu'à ce que la plaque de croissance se résorbe, Cbfal n'est requis que pendant le développement et donc vraisemblablement seulement au démarrage du processus de maturation des chondrocytes puisque l'expression de ce gène devient indétectable dans ces cellules aprés la naissance $[10,47]$.

Enfin, un autre facteur de transcription, Msx2, est lui vraisemblablement impliqué dans la régulation du processus de prolifération des chondrocytes plutôt que de leur maturation

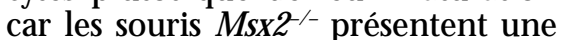
réduction globale de l'épaisseur des cartilages de croissance due à une diminution du nombre total de chondrocytes [9]. Son rôle moléculaire reste néanmoins à préciser car seule une réduction mineure de l'expression de PTHrP a été décelée chez ces souris, réduction qui à elle seule ne permet pas d'expliquer clairement leur phénotype.

\section{Troisième étape : vascularisation, résorption des modèles cartilagineux et ostéogenèse}

A ce stade de la squelettogenèse, les os en formation peuvent être identifiés par une juxtaposition en miroir des deux plaques de croissance (figure 2). Au fur et à mesure du développement, ces structures régresseront vers les deux extrémités du futur os jusqu'à n'en occuper qu'une région très restreinte. Ces deux zones cartilagineuses seront responsables de la croissance longitudinale des os en période post-natale. Elles ne se résorberont qu'à la puberté, entraînant l'arrêt de la croissance.

Une fois leur maturation achevée, les chondrocytes hypertrophiques sont encastrés dans une matrice calcifiée enrichie notamment en collagène de type $X$. O utre la synthèse de cette matrice, un élément important du programme génétique propre aux chondrocytes hypertrophiques est la capacité de sécrétion du VEGF (vascular endothelial growth factor) [49]. Le VEGF est un puissant facteur angiogénique dont l'expression dans les chondrocytes est sous le contrôle de Cbfal [50]. L'haplo-insuffisance en VEGF étant létale très tôt au cours du développement (E11-E12), ce sont 
des expériences d'administration post-natale d'un récepteur soluble inactivant le VEGF endogène [49] et d'inactivation spécifique du gène dans les chondrocytes [51] qui ont permis de caractériser son importance dans le processus endochondral. En l'absence d'activité VEGF, bien que toute la cascade de différenciation des chondrocytes s'accomplisse normalement, la formation des os va être défectueuse. (1) Les ostéoblastes, qui expriment le récepteur du VEGF à leur surface, ne migrent pas du bone collar vers les centres hypertrophiques des condensations par chimiotactisme et le dépôt de matrice osseuse ne se produit pas. (2) En l'absence de vascularisation, la migration des ostéoclastes dans les modèles cartilagineux ne peut se faire non plus. En effet, c'est par voie vasculaire que transitent les précurseurs des ostéoclastes, ces cellules multinucléées d'origine myéloïde capable de résorber la matrice osseuse pour permettre le modelage et le remodelage de ce tissu rigide. En leur absence, la matrice cartilagineuse calcifiée déposée par les chondrocytes hypertrophiques ne pourra pas être digérée afin d'être remplacée par une matrice osseuse synthétisée par les ostéoblastes.

Deux facteurs, Ihh et Cbfal, dont on a déjà vu l'importance dans la maturation des chondrocytes, interviennent aussi dans le contrôle de la différenciation des ostéoblastes. En effet, c'est en réponse à la production d'Ihh par les chondrocytes hypertrophiques que les cellules mésenchymateuses situées à la périphérie de la condensation vont commencer à se différencier en ostéoblastes, individualisant histologiquement le bone collar par leur capacité de synthétiser une matrice osseuse [45]. Comme nous l'avons vu, des souris chimères sauvages comportant des cellules PPR ${ }^{-1-}$ présentent des chondrocytes pré-hypertrophiques (comme hypertrophiques) ectopiques [52]. Si ces cellules sont par ailleurs normales, l'expression d'Ihh y sera induite et des bone collar se formeront de manière ectopique. Si en revanche ces cellules sont également $\mathrm{Ihh}^{-1-}$, il n'y aura pas de formation ectopique de bone collar. En fait, il est possible qu'en l'absence d'Ihh les cellules du bone collar soient incapables de se différencier en ostéoblastes parce que
Cbfal n'y est pas exprimé [45]. En effet, Cbfal est un activateur obligatoire de la différenciation ostéoblastique. Ce facteur de transcription a été à l'origine isolé grâce à sa capacité de régler l'expression de gènes spécifiquement exprimés dans les ostéoblastes [10]. En son absence, la différenciation ostéoblastique ne se fait pas, ni selon le mode intramembranaire ni selon le mode endochondral, et le squelette des souris reste entièrement cartilagineux [10]. Le rôle joué par Cbfal sur la différenciation ostéoblastique est complètement distinct de celui qu'il a lors de l'hypertrophie des chondrocytes. En effet, des souris $\mathrm{Cbfa}^{-1-}$ dans lesquelles la présence de Cbfal est restaurée dans les chondrocytes mais pas dans les cellules ostéoprogénitrices du bone collar présentent une absence de différenciation ostéoblastique alors que toutes les autres étapes de la squelettogenèse, hypertrophie, vascularisation et ostéoclastogenèse peuvent se produire normalement [47].

Enfin, il a tout dernièrement été proposé que le basculement entre prolifération et différenciation des cellules pré-ostéoblastiques soit contrôlé par $\mathrm{pRb}$, un facteur anti-tumoral clé qui règle le cycle cellulaire dans de nombreux types de cellules. pRb exercerait en outre un effet différenciateur dans les ostéoblastes en interagissant avec Cbfal pour activer l'expression des gènes caractéristiques de ce processus [53]. Cette hypothèse est confortée in vivo par le fait que chez les patients haploinsuffisants pour l'expression de $\mathrm{pRb}$, le second type de tumeur observé après les rétinoblastomes sont les ostéosarcomes et que dans $60 \%$ des cas d'ostéosarcomes sporadiques, il y a déficience du gène codant pour pRb [54].

\section{Perspectives}

Les données présentées dans cet article illustrent bien la puissance des analyses génétiques quand il s'agit de disséquer des mécanismes complexes de régulation. En effet, les interactions qui se passent par exemple entre chondrocytes de la plaque de croissance et les ostéoblastes du bone collar auraient été très difficiles à déceler si des modèles in vivo n'avaient pas été utilisés. A noter également que dans la majorité des cas où la comparaison a pu être faite, les mêmes facteurs remplissent les mêmes fonctions chez I'homme et chez la souris, validant I'utilisation de ce modèle dans l'étude de la squelettogenèse. II est donc prévisible que ce type d'étude va se multiplier dans les années à venir et que d'autres facteurs vont être identifiés, puis leur fonction et leurs interactions avec les régulateurs déjà connus caractérisées. Il est néanmoins important de souligner que les études purement génétiques non seulement n'excluent pas mais nécéssitent des études complémentaires soit biochimiques, soit d'analyse de régulation transcriptionnelle ou post-translationnelle. Ces études seront précieuses notamment pour clarifier le caractère direct ou mettre en évidence l'existence d'intermédiaires au sein d'une cascade génétique connue

\section{RÉFÉRENCES}

1. Zakany J, Duboule D. H ox genes in digit development and evolution. Cell Tissue Res 1999 ; 296 : 19-25

2. OIsen BJ, Reginato AM, Wang W. Bone Development. Annu Rev Céll Dev Biol 2000; 16: $191-220$

3. Karsenty G. Genetics of skeletogenesis. Dev Genet 1998; 22: 301-13.

4. Min $\mathrm{H}$, Danilenko $M$, Scully $S$, et al. Fgf-10 is required for both limb and lung development and exhibits striking functional similarity to Drosophila branchless. Genes Dev 1998; 12: 3156-61.

5. Hogan BLM. Bone morphogenetic proteins: multifunctional regulators of vertebrate development. Genes Dev 1996; 10: 1580-94.

6. Satokata I, Maas R. MsxI deficient mice exhibit cleft palate and abnormalities of craniofacial and tooth development. Nat Genet $1994 ; 6$ : 348-55.

7. van den Boogaard MJ, Dorland M, Beemer FA, van Amstel HK. MSX 1 mutation is associated with orofacial clefting and tooth agenesis in humans. Nat Genet 2000; 24 : 342-3.

8. Peters $\mathrm{H}$, Wilm E, Sakai N, Imai K, Maas $R$, Balling R. Pax1 and Pax9 synergistically regulate vertebral column development. Development 1999; 126: 5399-408.

9. Satokata I, Ma L, Ohshima H, et al. M sx2 deficiency in mice causes pleiotropic defects in bone growth and ectodermal organ formation. Nat Genet $2000 ; 24: 391-5$. 


\section{RÉFÉRENCES}

10. Ducy P. Cbfal: a molecular switch in osteoblast biology. Dev Dyn 2000; 219: 46171.

11. Wilkie AOM. Craniosynostosis: genes and mechanisms. Hum Mol Gen 1997; 6: 1647-56.

12. Zhou YX, Xu X, Chen L, Li C, Brodie SG, Deng CX. A Pro250Arg substitution in mouse Fgfrl causes increased expression of $\mathrm{Cbfal}$ and premature fusion of calvarial sutures. H um M ol Gen 2000; 9: 2001-8.

13. El Ghouzzi V, Le Merrer M, PerrinSchmitt $F$, et al. Mutations of the Twist gene in the Saethre-Chotzen syndrome. Nat Gen et 1997: $15: 42-6$

14. H oward TD, Paznekas WA, Green ED, et al. Mutations in TWIST, a basic helix-loophelix transcription factor in Sathre-Chotzen syndrome. Nat $\mathrm{G}$ en et $1997 ; 15$ : 36-41.

15. Rice DPC, Aberg T, Chan YS, et al. Integration of FGF and TWIST in calvarial bone and suture development. Development 2000 ; $127: 1845-55$

16. Jabs EW, Muller U, Li X, et al. A mutation in the homeodomain of the human MSX 2 gene in a family affected with autosomal dominant craniosynostosis. Cell 1993 575 : 443-50.

17. Liu YH, Kundu E, Wu L, et al. Premature suture closure and ectopic cranial bone in mice expressing M sx2 trransgene in the developing skull. Proc Natl Acad Sci USA 1995; 92 : 6137-41.

18. Lefebvre $V$, de Crombrugghe B. Toward understanding SOX 9 function in chondrocyte differentiation. M atrix Biol 1998; 16: 529-40.

19. Bi W, Deng JM, Zhang Z, Behringer $R$ de Crombrugghe B. Sox9 is required for cartilage formation. N at Gen et 1999; 22: 85-9.

20. Smits P, Li P, Mandel I et al. The trans cription factors L-Sox5 and Sox6 are essential for cartilage formation. Dev Cell 2001; 1 : 277-90.

21. Colnot C, Sidhu SS, Balmain N, Poirier F. Uncoupling of chondrocyte death and vascular invasion in mouse galectin 3 null mutant bones. Dev Biol 2001; 229: 203-14.

22. Vajo Z, Francomano CA, Wilkin DJ. The molecular and genetic basis of fibroblast growth factor receptor 3 disorders: the achondroplasia family of skeletal dysplasia, Muenken craniosynostosis, and Crouzon syndrome with acanthosis nigricans. Endocrinol Rev 2000; 21: 23-39.

23. Nasaki MC, Wang O, Xu J, Ornitz DM. Graded activation of fibroblast growth factor receptor 3 by mutations causing achondroplasia and thanatophoric dysplasia. $\mathrm{N}$ at Gen et 1996; 13: 233-6.

24. Coffin JD, Florkiewicz RZ, Neumann J, et al. Abnormal bone growth and selective growth factor (FGF-2) transgenic mice. Mol Biol Cell 1995; 6: 1861-73.

25. Garofalo S, Kliger-Spatz M, Cooke JL, et al. Skeletal dysplasia and defective chondrocyte differentiation by targeted overexpression of fibroblast growth factor 9 in transgenic mice. J Bone M iner Res 1999; 14 : 1909-15.

26. Colvin $\mathrm{S}$, Bohne BA, Harding GW, McEwen DG, Ornitz DM. Skeletal overgrowth and deafness in mice lacking fibroblast growth factor receptor 3 . Nat G en et 1996 ; 12 : 390-7.

27. Deng C, Wynshaw-Boris A, Zhou F, Kuo $A$, Leder P. Fibroblast growth factor receptor 3 is a negative regulator of bone growth. Cell 1996; 84: 911-21.

28. Chen L, Adar R, Yang X, et al. Gly369Cys mutationin mouse FGFR3 causes achondroplasia by affecting both chondrogenesis and osteogenesis. J Clin Invest 1999; 104: 1517-25.

29. Su WCS, Kitagawa M, Xue N, et al. Activation of Statl by mutant fibroblast growthfactor receptor in thanatophoric dysplasia type II dwarfism. Nature 1997; 386: 288-92.

30. Sahni M, Ambrosetti DC, Mansukhani A, Gertner R, Levy D, Basilico C. FGF signaling inhibits chondrocyte proliferation and regulates bone development through the STAT-1 pathway. Genes Dev 1999; 13: 1361-6.

31. I wata $T$, Chen $L$, Li C, et al. A neonatal lethal mutationin FG'FR3 uncouples proliferation and differentiation of growth plate chondrocytes in embryos. H um $\mathrm{M}$ ol $\mathrm{Gen}$ $2000 ; 9$ : 1603-13.

32. Segev O, Chumakov I, Nevo Z, et al. Restrained chondrocyte proliferation and maturation with abnormal growth plate vascularization and ossification in human FGFR-3G380R transgenic mice. $\mathrm{H}$ um $\mathrm{M}$ ol $\mathrm{Gen}$ 2000; 9 : 249-58.

33. I wata T, Li C, Deng CX, Francomano CA. Highly activated Ffgr3 with the K644M mutation causes prolonged survival in severe dwarf mice. H um M ol Gen 2001; 10 : $1255-64$.

34. Karaplis AC, Luz A, Glowacki J, et al. Lethal skeletal dysplasia from targeted disruption of the parathyroid hormone-related peptide gene. Genes Dev 1994 ; 8: 277-89.

35. Weir EC, Philbrick WM, Amling M, N eff $L A$, Baron $R$, Broadus AE. Targeted overexpression of parathyroid hormone-related peptide in chondrocytes causes chondrodysplasia and delayed endochondral bone formation. Proc Natl Acad Sci USA 1996; 93: 10240-5.

36. Lanske $B, K$ araplis $A C$, Lee $K$, et al. PTH / PTH rP receptor in early development and Indian hedgehog-regulated bone growth. Science 1996; 273: 663-6.

37. Vortkamp A, Lee K, Lanske B, Segre GV, Kronenberg HM, Tabin CJ. Regulation of rate of cartilage differentiation by Indian $\mathrm{H}$ edgehog and PTH -Related protein. Science 1996; 273: 613-22.

38. Hock JM. Anabolic actions of PTH in the skeletons of animals. J M usculoskel Neuron Interact $2001 ; 2$ : 33-47.

39. Lanske $B$, Amling M, Neff LA, Guiducci Baron $R$, Kronenberg HM. Ablation of the PTH rP gene or the PTH/ PTHrP receptor gene leads to distinct abnormalities in bone development. J Clin Invest 1999; 104 : 399-407.

40. Jobert AS, Zhang $P$, Couvineau $A$, et al. Absence of functional receptors for parathyroid hormone and parathyroid hormonerelated peptide in blomstrand chondrodysplasia. J Clin Invest 1998; 102: 34-40.

41. Karaplis AC, He B, Nguyen MTA, et al. Inactivating mutation in the human parathyroid hormone receptor type I gene in blomstrand chondrodysplasia. Endocrinology 1998; 139: 5255-8.

42. Schipani E, Kruse K, Juppner H. A constitutively active mutant PTH-PTH rP receptor in Jansen-type metaphyseal chondrodysplasia. Science 1995; 268: 98-100.

43. Schipani E, Lanske B, Hunzelman J, et al. Targeted expression of constitutively active receptors for parathyroid hormone and parathyroid hormone-related peptide delays endochondral bone formation and rescues mice that lack parathyroid hormone-related peptide. Proc Natl Acad Sci USA 1997; 94 : 13689-94.

44. Chung UI, Lanske B, Lee K, Li E, Kronenberg $\mathrm{HM}$. The parathyroid hormone/ parathyroid hormone-related peptide receptor coordinates endochondral bone development by directly controlling chondrocyte differentiation. Proc Natl Acad Sci USA 1998; 95 : 13030-5.

45. St-Jacques $B, H$ ammerschmidt $M$, McMahon AP. Indian hedgehog signaling regulates proliferation and differentiation of chondrocytes and is essential for bone formation. Genes Dev 1999; 13 : 2072-86.

46. Karp SJ, Schipani R, St-Jacques B, Hunzelman I, Kronenberg $\mathrm{H} M$. Indian $\mathrm{H}$ edgehog coordinates endochondral bone growth and morphogenesis via parathyroid hormone related-protein-dependent and independent. Development 2000; 127: 543-8.

47. Takeda S, Bonnamy JP, O wen MJ, Ducy $P$, Karsenty $G$. Continuous expression of Cbfal in non-hypertrophic chondrocytes uncovers its ability to induce hypertrophic chondrocytes differentiation and partially rescues Cbfal-deficient mice. Genes Dev $2001 ; 15$ : 467-81.

48. U eta $C$, I wamoto $M$, Kanatani N, et al. Skeletal malformations caused by overexpression of Cbfal or its dominant negative form in chondrocytes. J Cell Biol 2001; 153: 87-99.

49. Gerber HP, Vu T, Ryan AM, Kowalski J, Werb Z, Ferrara N. VEGF couples hypertrophic cartilage remodeling, ossification and angiogenesis during endochondral bone formation. Nat M ed 1999; 5 : 623-8.

50. Zelzer E, Glotzer DJ, Hartmann C, et al. Tissue specific regulation of VEGF expression during bone development requires Cbfa1/ Runx2. M ech Dev 2001; 106: 97-106. 


\section{RÉFÉRENCES}

51. Haigh If, Gerber H P, Ferrara N, Wagner $\mathrm{EF}$. Conditional inactivation of VÉGF-A in areas of collagen2al expression results in embryonic lethality in the heterozygous state. Development 2000; 127 : 1445-53.

52. Chung UI, Schipani E, MCMahon AP, Kronenberg H'M. Indian hedgehog couples chondrogenesis to osteogenesis in endochondral bone development. J Clin Invest 2001; 107: 295-304.

53. Thomas DM, Carty SA, Piscopo DM, et al. The retinoblastoma protein acts as a transcriptional coactivator required for osteogenic differentiation. $\mathrm{M}$ ol Cell 2001; 8: 303-16.

54. Hansen MF, Koufos A, Gallie BL, et al. O steosarcoma and retinoblastoma: a shared chromosomal mechanism revealing recessive predisposition. Proc Natl Acad Sci USA 1985; 82: 6216-20.

\section{Summary}

Genetic control of skeletogenesis

Thanks to the progresses of human genetics and to the explosion of genome manipulations in mice, our understanding of skeletogenesis has made tremendous advances during the last 10 years. For instance, growth factors and transcription factors have been identified that trigger and regulate the differentiation of the 3 cell types specific to the skeleton: chondrocytes, osteoblasts and osteoclasts. Furthermore, in several instance, genetic cascades can now be drawn that integrate both cellular and structural events during skeletogenesis. This review will summarize the most recent advances of the field of skeleton development, trying to present a comprehensive view of the steps and regulatory events that genetically control skeletogenesis.
TIRÉS À PART

P. Ducy.

$\mathrm{m} / \mathrm{s} n^{\circ} 12$, vol. 17, décembre 2001

\section{4 ${ }^{\text {es }}$ Journées de Biologie Clinique Necker - Institut Pasteur Institut Pasteur, Paris - 21-23 janvier 2002}

Présidées par le Pr Pierre Kamoun (Biochimie, Necker), les 44s Journées de Biologie Clinique Necker - Institut Pasteur comprendront deux journées de conférences (21-22 janvier), au Centre d'Information Scientifique de l'Institut Pasteur, et une journée de démonstrations pratiques (le 23 janvier), dans les laboratoires de l'Hôpital Necker et de l'Institut Pasteur.

Quelque 250 participants - biologistes hospitaliers, biologistes privés, industriels - y sont attendus.

\section{Thèmes au programme}

Des conférences (21-22 janvier)

- Évolution des concepts de l'auto-immunité et de la détection des auto-anticorps

- Génétique et cancer

- Génétique et infertilité

- Actualités sur le Purpura Thrombopénique Idiopathique (PTI)

- Renouveau des images microscopiques numérisées en hématologie

- Les nouveaux antithrombotiques

- Les anémies macrocytaires

- Immunoglobulines monoclonales - Diagnostic biologique

- Nouveaux immuno-suppresseurs

- Les marqueurs du remodelage osseux

- Exploration des lipides sériques : les examens d'aujourd'hui et de demain

- Légionelloses

- Helicobacter pylori et cancers gastriques

- Listeria

- Biologie moléculaire en virologie

Des démonstrations pratiques (23 janvier)

- Cas cliniques d'hématologie

- Difficultés diagnostiques en hémobiologie (groupes sanguins, RAI)

- Staphylocoques

- Diagnostic biologique de la grippe

Rappelons que, depuis leur création en 1958 par le Pr Jean Hamburger, ces Journées aident la recherche en finançant une bourse d'études à un jeune chercheur du CHU Necker ou de l'Institut Pasteur.

Renseignements et inscriptions: Isabelle Van Laethem, DVL, 101, rue Mademoiselle, 75015 Paris. Tél. : 0145665342 - Fax: 0147834488. E-mail:dvl@wanadoo.fr 